\title{
Rearrangement and expression of myeov and hst in NIH/3T3 transfectants: A caveat for the interpretation of DNA transfection analyses
}

\author{
MARCUS BRECHT, AGATHA C.M. STEENVOORDEN, SUSANNE LUF, \\ CLAUS R. BARTRAM and JOHANNES W.G. JANSSEN
}

Institut fuer Humangenetik, Universitaetsklinikum Heidelberg, Im Neuenheimer Feld 366, D-69120 Heidelberg, Germany

Received October 20, 2006; Accepted November 24, 2006

\begin{abstract}
By means of the tumorigenicity assay applying DNA from a patient with a gastric carcinoma (MA) we have already reported the identification of the putative oncogene myeov. In addition we have shown its involvement in $\mathrm{t}(11 ; 14)$ positive multiple myelomas and amplifications of breast tumours and esophageal carcinomas. The failure of myeov cDNA to induce tumour formation in $\mathrm{NIH} / 3 \mathrm{~T} 3$ cells prompted us to analyze the human sequences present in our MA-T1a1 tertiary transfectants. Sequence analysis revealed the presence of the human oncogene hst ( $f g f 4)$ at a distance of $\sim 9 \mathrm{~kb}$ from the myeov gene in our MA-T1a1 tertiary transfectants. Both myeov and hst (fgf4) are normally situated $\sim 475-\mathrm{kb}$ apart at band $11 \mathrm{q} 13$, a region that is frequently amplified and overexpressed in various tumours. Southern and Northern blot analyses confirmed our sequence data and showed rearrangement of $h s t$ sequences during the transfection process and its expression in our MA-T1a1 tertiary transfectants.
\end{abstract}

\section{Introduction}

Using the tumorigenicity assay our group was able to identify and/or isolate a plethora of different oncogenes, such as mas, ufo, p85//humorf8, ret, cot, tre, hst (fgf4), dbl, B-raf, gef-hl and myeov (1-5). This latter gene, myeov, was identified by the tumorigenicity assay using DNA from the tumour of a patient with gastric carcinoma and assigned to the chromosomal region $11 \mathrm{q} 13$. The $11 \mathrm{q} 13$ region is involved in genomic/chromosomal rearrangements in a variety of human malignancies, including reciprocal translocations in B-cell neoplasms, unbalanced translocations or chromosomal inversions and frequent DNA amplification in various carcinomas, such as breast tumours, head and neck cancers,

Correspondence to: Dr Johannes W.G. Janssen, Institut fuer Humangenetik, Universitaetsklinikum Heidelberg, Im Neuenheimer Feld 366, D-69120 Heidelberg, Germany

E-mail: hans_janssen@med.uni-heidelberg.de

Key words: NIH/3T3 transformation assay, myeov, hst (fgf4), cancer, $11 \mathrm{q} 13$ oesophageal squamous cell carcinomas (ESC) and many other types of solid tumours (1). We were able to show that myeov is rearranged and activated concomitantly with cyclinD1 $(C C N D 1)$ in a subset of human $\mathrm{t}(11 ; 14)(\mathrm{q} 13 ; \mathrm{q} 32)$-positive multiple myeloma cell lines (1). In a collaborative study, we also showed that myeov is frequently coamplified with cyclinD1 (9.5\%) and abnormally expressed (16\%) in a number of human breast carcinomas (6). Moreover we elucidated the possible involvement of myeov in ESC carcinogenesis and found that myeov was coamplified with cyclinDl in a great number of cell lines and primary tumours tested. However, myeov RNA overexpression was only detected in a subset of cell lines carrying myeov amplification, a phenomenon attributed to aberrant methylation of the myeov promoter (7). Zoo blot analysis and database analyses revealed that the myeov gene is present in humans and chimpanzee, but not in Zebrafish, frog, dog, sheep, mouse or rat. Myeov mRNA expression has only been detected in humans. A computer search with the deduced MYEOV protein sequence did not detect any homology with known protein motifs or domains. Its exact cellular function therefore remains elusive.

The $h s t / f g f 4$ gene was originally identified as a transforming gene in DNAs from gastric carcinomas, a noncancerous portion of gastric mucosa and a Kaposi sarcoma by transfection assays using NIH/3T3 cells (8-10). Expression of its cDNA in an expression vector and the genomic hst gene induced the transformation of NIH/3T3 cells upon transfection $(11,12)$. The mouse homologue of the hst/fgf4 gene has been found to be activated by proviral insertion in some virally induced mammary tumours (13).

Despite the detection of human myeov DNA sequences and myeov RNA expression in our MA-transfectants, its involvement in $\mathrm{t}(11 ; 14)$ translocations in multiple myelomas and its amplification and overexpression in various carcinomas, we were not able to transform NIH/3T3 cells using myeov cDNA. In order to clarify the exact constellation in the thirdcycle transfectant surrounding the myeov gene we determined the complete sequence of the alu-positive phage clone.

\section{Materials and methods}

Cell lines. The murine fibroblastic cell line (NIH/3T3) was originally obtained from M. Wigler (14). The cells utilized in transfection assays (see below) were derived from a single 
clone $\left(\mathrm{B}_{2} 5\right)$ selected by us on the basis of flat morphology, low incidence of spontaneous overgrowth, and ability to transform efficiently with T24 bladder carcinoma DNA containing an activated $H$-ras gene (15). NIH/3T3 cells were propagated in Dulbecco's modified Eagle's medium supplemented with $10 \%$ NCS and antibiotics. Cell lines were propagated in a humidified $\mathrm{CO}_{2}$ incubator with $5-7 \% \mathrm{CO}_{2}$ at $37^{\circ} \mathrm{C}$.

Transfection assay. High molecular weight DNA of a stomach cancer was cotransfected into NIH/3T3 cells together with a dominant drug-resistant selectable marker, pRSVneo, followed by G418 selection and injection of the resulting colonies into nude mice, as described (16).

Genomic cloning. DNA isolated from a third-cycle induced mouse tumour MA-T1A1 was partially digested with Sau3AI, treated with calf intestine phosphatase, fragments fractionated by size using $\mathrm{NaCl}$ or sucrose gradients, ligated to phage (EMBL-3) arms (17), packaged in vitro, plated and screened with a human alu probe $(4,18)$. Genomic subfragments of an alu-positive phage were sub-cloned into digested pT7T3 plasmid DNA (Amersham Pharmacia Biotech, Freiburg, Germany).

DNA and RNA purification and analysis. High molecular weight DNA from cell lines or phage clones was prepared according to standard methods. DNA was digested with restriction endonucleases, electrophoresed on a $0.6 \%$ agarose gel and blotted onto Nytran 13N membranes (Schleicher and Schuell, Dassel, Germany). Filters were hybridized in 3X SSC (0.45 M NaCl, 0.045 M sodium citrate), 5X Dennhardt's, $200 \mu \mathrm{g} / \mathrm{ml}$ denatured salmon sperm DNA, 1\% SDS and 10\% dextran sulphate at $63^{\circ} \mathrm{C}$ for $16 \mathrm{~h}$ with randomly primed ${ }^{32} \mathrm{P}$ labelled probes (MBI Fermentas, St. Leon-Rot, Germany) with $2 \mu \mathrm{g} / \mathrm{ml}$ pRSVneo DNA as competitor DNA to avoid cross-hybridization with cotransfected pRSVneo sequences. Subsequently the filters were extensively washed in 3X SSC, $0.1 \%$ SDS at $63^{\circ} \mathrm{C}$, followed by a moderate or high stringency wash. Filters were exposed to Kodak X-Omat DS, XAR-5, or MS-film at $-70^{\circ} \mathrm{C}$ with Ilford intensifier or MS screens (5). For hybridization analysis we used the following insert probes; 300-bp insert from the human repetitive specific alu plasmid Blur-8 (19), a 285-bp SacI-HindIII fragment from the pORF1 plasmid containing hst cDNA, a 5' EcoRI myeov cDNA fragment and a rat GAPDH cDNA sequence. Total RNA was isolated and purified by acid guanidinium isothiocyanate/phenol-chloroform extraction, as described (20). PolyA ${ }^{+}$RNA was purified using an Oligotex mRNA mini kit (Qiagen GmbH, Hilden, Germany) per the manufacturer's instructions. Northern blot analyses were performed as described by Shackleford and Varmus (21). Briefly, comparable amounts of polyA ${ }^{+}$RNA were resolved on $1.2 \%$ agarose formaldehyde gels, blotted on to Hybond-N membranes (Amersham Pharmacia Biotech) and then hybridized as described for Southern blotting.

DNA sequencing. Plasmids containing large inserts were sequenced by application of the EZ::TN ${ }^{\mathrm{TM}}<\mathrm{TET}-1>$ insertion system, based on the Tn5 transposition system (22). This system permits high efficiency, random insertion of an
EZ::TN ${ }^{\mathrm{TM}}$ Transposon into DNA target molecules in vitro and accelerates sequencing of large DNA templates. As the primers supplied by the manufacturer (TET-1 FP-1 and RP-1) failed to provide the expected results, we developed a new primer set (TET-1 rev 2:5' IRD700-gtaccgagctcgaattcatcg 3' and TET-1 FP-2:5' IRD800-gtcgacctgcaggcatgc 3') from the EZ::TN ${ }^{\mathrm{TM}}<\mathrm{TET}-1>$ Transposon sequence that gave good results using a LI-COR long Readir 4200.

Plasmid miniprep DNA samples were sequenced with a Reader $^{\text {TM }}$ Taq polymerase fluorescent-labelled primer CycleReader $^{\mathrm{TM}}$ auto DNA sequencing kit with 7-deaza-dGTP (MBI Fermentas) on a LI-COR long Readir 4200 DNA sequencer.

Computer search and programs. Sequences were evaluated with the University of Wisconsin Genetics Computer Group sequence analysis package (version 10.0-Unix) at HUSAR in the DKFZ Heidelberg, Germany and with server facilities of the National Center for Biotechnology Information, National library of Medicine, National Institute of Health, Bethesda, MD, USA (www.ncbi.nlm.nih.gov), with the Ensemble program of EMBL-EBI and the Sanger Institute supported by the Wellcome Trust (www.ensembl.org), as well as with the NIX program at the UK HGMP Resource Center or MRC Rosalind Franklin Centre for Genomics Research (RFCGR) (www.hgmp.mrc.ac.uk).

\section{Results}

Using DNA from a gastric carcinoma (MA) in the NIH/3T3transformation/tumorigenicity assay we were able to induce tumours in nude mice with a latency period of 6 weeks. Southern blot analysis of DNA obtained from a tertiary nude mouse tumour exhibited a consistent pattern of human alu repeat sequences after digestion with the restriction enzyme EcoRI (Fig. 1). DNA of a tertiary transfectant was cloned into the EMBL-3 phage vector and probed for human sequences using human alu-repetitive sequences. One single alu-positive phage clone was isolated. Exon-trap analysis of genomic subfragments from this alu-positive phage clone revealed a 400-bp exon-trap fragment that was used for Northern blot analysis and detected two transcripts in RNA of a third-cycle transfectant (MA-T1A1) but not in normal $\mathrm{NIH} / 3 \mathrm{~T} 3$ cells. cDNA cloning enabled the cloning of a novel putative transforming gene called myeov (1). In addition we showed overexpression of myeov as a consequence of a specific chromosomal translocation in multiple myelomas (1) and amplification and overexpression of myeov in oesophageal squamous cell carcinomas (ESC) (7) and breast tumours (6). Although these data convincingly support a causal role for myeov in NIH/3T3 transformation and cancer, we were not able to confirm its potency to elicit a transformed phenotype in NIH/3T3 cells by means of overexpression of full-length myeov cDNA. To resolve this apparent contradiction we decided to determine the complete sequence of the only alupositive phage clone of the third-cycle transfectant (MAT1A1) that we were able to isolate. In addition to the presence of myeov sequences, database analysis revealed the presence of hst sequences in this alu-positive phage clone (Fig. 2). As mentioned above, hst was detected by the NIH/3T3 


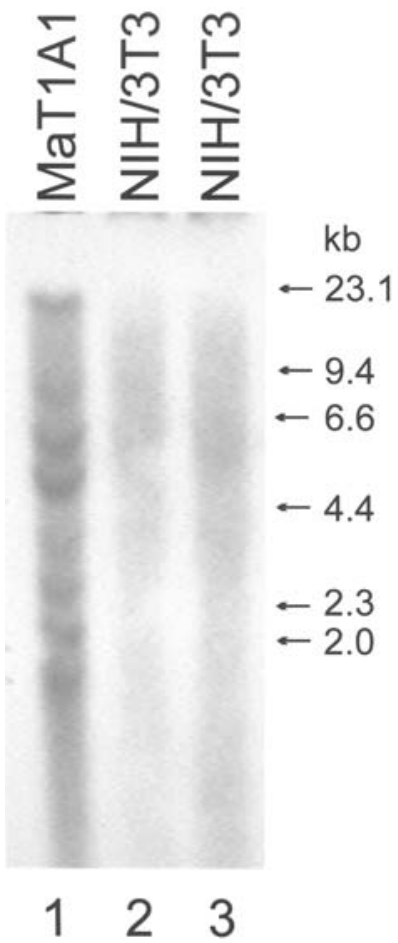

Figure 1. Southern blot analysis of the MA-T1a1 transfectant. DNAs of the tertiary MA-T1a1 transfectant and of control NIH/3T3 cells were digested with EcoRI, blotted and hybridized to human alu-sequences. $\lambda$-HindIII DNA fragments served as molecular-weight markers and are indicated at the right margin.

transformation assay using DNAs from different tumours $(8,9)$. The transforming capacity of $h s t$ in NIH/3T3 cells has been proven $(10,11)$. Both myeov and hst are located on chromosome 11 band q13.1 at a distance of $\sim 475 \mathrm{~kb}$. In the MA-T1A1 tertiary transfectants two fragments of the 11q13.1 region (AP005232.3 and AP006345.3) containing the myeov and hst genes are joined, respectively. In this situation the myeov gene DNA and $h s t$ gene DNA are located at a distance of $\sim 9 \mathrm{~kb}$ from each other.

To resolve the mechanism of activation of the hst ( $f g f 4$ ) gene and to confirm our sequence data, we performed Southern blot analysis. DrdI-digested DNA from two healthy individuals, the original stomach tumour as well as DNA from a tertiary MA-T1 transfectant and NIH/3T3 control cells was hybridized with a probe containing the $3^{\prime}$ portion of hst (fgf4) cDNA (Fig. 3). In the two healthy individuals and in the original gastric carcinoma, hst (fgf4) hybridised with a DrdI fragment of $\sim 11 \mathrm{~kb}$, which exactly corresponds to the original genomic constellation in the ensemble database. DNA of our tertiary MA-T1a1 transfectant showed three bands, namely of $\sim 16.8 \mathrm{~kb}, \sim 8 \mathrm{~kb}$ and $\sim 1.8 \mathrm{~kb}$. The two smaller bands are also present in DNA of NIH/3T3 cells and therefore represent the murine homologue of the hst (fgf4) gene. The higher band of $\sim 16.8 \mathrm{~kb}$ represents rearranged human hst sequences in DNA of our MA-T1a1-transfectant and matched perfectly with our sequence data.

To confirm our data we performed a Northern blot analysis. RNA of the MA-T1a1-transfectant, an hst-negative transfectant and $h s t$-positive transfectant (5), both serving as controls were hybridized with the $h s t(f g f 4)$ probe. Our Northern blot
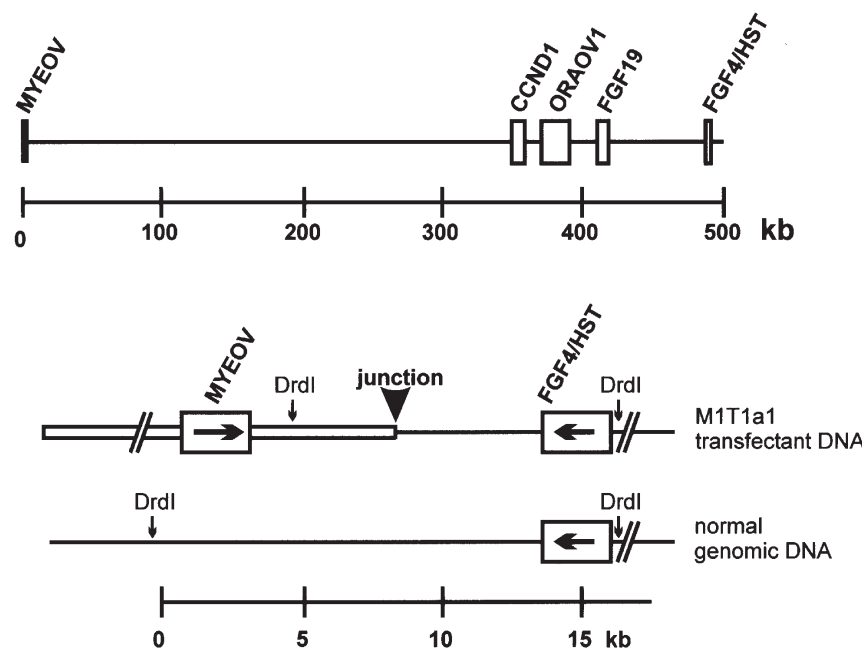

Figure 2. Genomic map illustrating the composition of human 11q13 sequences in MA-T1a1 transfectant DNA. In normal human DNA both myeov and hst (fgf4) genes are situated on chromosome 11 in the region q13 and are separated by $\sim 475 \mathrm{~kb}$ (upper part). The relative positions of the different genes on 11q13 are indicated by boxes. Sequence data revealed that in DNA of the MA-T1a1 transfectant two fragments of human chromosome $11 \mathrm{q} 13$ containing myeov and hst (fgf4) were present. The distance between myeov and hst (fgf4) in MA-T1a1 DNA was $\sim 9 \mathrm{~kb}$. DrdI restriction sites in MA-T1a1- and normal-DNA are indicated. The junction between the two fragments from human chromosome 11q13 is indicated by a filled arrow.

data revealed $h s t$ ( $f g f 4$ ) transcripts in RNA of the $h s t$-positive transfectant as well as in RNA of the MA-T1a1 transfectant and thus confirmed our Southern blot and sequence data. RNA of the MA-T1a1 transfectant shows the expected and previously described myeov transcripts (1).

\section{Discussion}

The application of the NIH/3T3 transformation assay and its variants has allowed us and others to detect several oncogenes exhibiting diverse functions. Except for the ras genes most genes became activated by truncation, loss of gene or regulatory sequences or gene fusion. In some cases the oncogenes were already activated in the original tumour prior to gene transfer, i.e. the ras genes in various neoplasias, the met gene in the MNNG-HOS cell line (23), the erbB2 (formerly $n e u)$ in ENU-induced rat neuroblastomas (24) and the trk oncogene in a human colon carcinoma (25).

The presence of unrelated DNA fragments originating from different regions of the human genome in tumour/ transfectant DNAs has already been reported by us and others, i.e. $g e f-h l(2)$ and $m c f-2$, an activated form of the $d b l$ oncogene $(26,27)$. In these cases fragments derived from different chromosomes were joined together and integrated as a transgenome into the genome of murine NIH/3T3 cells. Considering the size of the human- and/or mouse genome, the fact that NIH/3T3 can take up $\sim 5000 \mathrm{~kb}$ of DNA by calcium phosphate transfection and that the size of purified genomic DNA fragments is $\sim 50-100 \mathrm{~kb}$, human DNA sequences present in tertiary NIH/3T3 transfectants should have a size of $\sim 50-80 \mathrm{~kb}$ and contain the activated human oncogene. An addition of the sizes of alu-positive fragments 


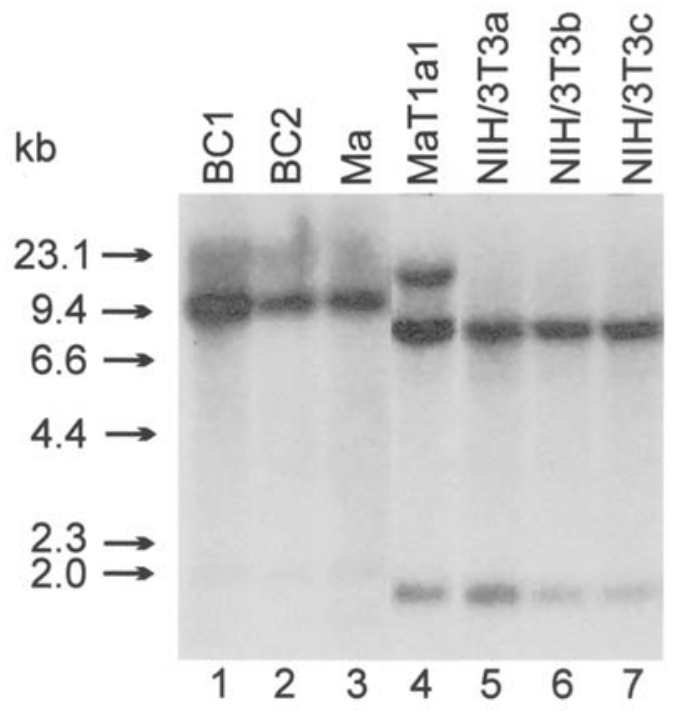

Figure 3. Southern blot analysis to confirm the recombination of hst (fgf4) sequences in MA-T1a1 DNA. DNAs from two healthy human individuals (BC1 and 2), the original human gastric tumour (MA), the MA-T1a1 tertiary transfectant and NIH/3T3 mouse control DNAs were digested with the restriction enzyme DrdI and hybridized with 3'-hst ( $f g f 4)$ cDNA sequences. $\lambda$-HindIII DNA fragments served as molecular-weight markers and are indicated at the left margin.

detected by Southern blot analyses in different NIH/3T3 transfectants supports this assumption (Fig. 1) (28). The complete sequence of a human transgenome, including its murine boundaries in a tertiary transfectant of the monocytic leukaemia cell line U937 proved that this assumption is correct (2). The myeov gene detected by us through application of the NIH/3T3 tumorigenicity assay to DNA from a gastric carcinoma possessed features that were also found for other oncogenes detected by this assay. The myeov gene was present on the human transgenome, highly expressed in its transfectants and not expressed in normal NIH/3T3 cells (1). In addition we showed that myeov was situated near a common $\mathrm{t}(11 ; 14)(\mathrm{q} 13 ; \mathrm{q} 32)$ breakpoint in multiple myelomas and showed overexpression in a subset of these $t(11 ; 14)$ positive multiple myeloma cell lines. Moreover we showed amplification and overexpression of myeov in breast cancer and in oesophageal squamous cell carcinomas $(6,7)$. Unfortunately we were not able to show any morphological transformation or tumour induction of NIH/3T3 cells transfected with myeov cDNA. In fact this prompted us to completely sequence an alu-positive phage clone obtained from tertiary MA-T1a1 transfectant DNA. Our sequence analysis revealed the presence of human $h s t$ ( $f g f 4$ ) sequences in the tertiary MA-T1a1 transfectant DNA. The hst/fgf4 gene was originally identified as a transforming gene in DNAs from gastric carcinomas, a noncancerous portion of gastric mucosa and a Kaposi sarcoma by transfection assays using NIH/3T3 cells (8-10). Our data therefore suggest that hst sequences were responsible for tumour induction in nude mice within our tertiary MA-T1a1 transfectants. It has been reported that genomic clones with $38-40 \mathrm{~kb}$ inserts and containing the hst (fgf4) gene of normal human DNA were capable of transforming NIH/3T3 cells. The loss of silencer or suppressor sequences located far from the coding sequence

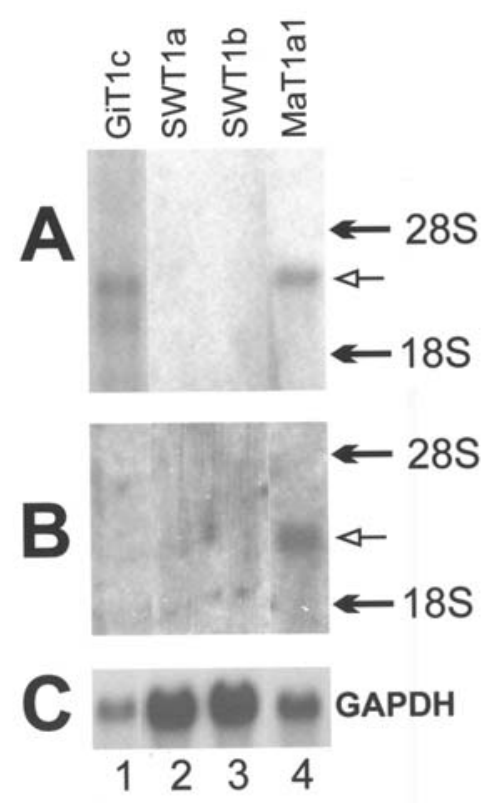

Figure 4. Detection of $h s t$ (fgf4) transcripts in MA-T1a1 transfectants. Two micrograms of poly $\mathrm{A}^{+}$RNA from an $h s t$-positive transfectant (GiT1c) (5), two different $h s t$-negative secondary transfectants (SWT1a, SWT1b) and MA-T1a1 were analyzed by Northern blot analyses. (A) Hybridization using a 3'-hst cDNA probe. (B) Hybridization using a 5'-myeov cDNA probe. Human hst transcript and myeov transcripts are indicated by open arrows. $28 \mathrm{~S}$ and $18 \mathrm{~S}$ ribosomal RNA were used as molecular weight markers and marked by closed arrows. (C) Control hybridization using a rat glyceraldehyde 3-phosphate dehydrogenase ( gapdh) probe.

would activate its expression $(29,30)$. We suggest a similar activation process in our MA transfectants.

On the other hand, it may be possible that myeov has an additive or synergistic effect. Both myeov and hst are located on 11q13, a frequent site of genetic aberration in a number of human malignancies, especially breast and head and neck cancers (31). Several candidate oncogenes have been proposed, i.e. myeov, cyclinD1, ems 1 and a recently characterized emsy gene in human breast cancer cell lines and primary tumours that binds to brca2 $(6,32,33)$. It is proposed that several genes are responsible for the amplifications at 11q13, because at least four distinct cores of amplification have been recognized and amplification profiles vary according to tumour type (33-35). In this study we report the joining of two fragments that are normally separated $\sim 475 \mathrm{~kb}$ in one transgenome in MA-T1a1 transfectants. Southern blot data revealed a band of similar size in DNA of two healthy individuals and the original stomach cancer implying that no rearrangement occurred in DNA of the original stomach tumour (Fig. 3, lanes 1-3). During transfection DNA fragments can and are randomly ligated to any other DNA fragment. The probability that two specific DNA fragments with a size of $\sim 40 \mathrm{~kb}$ are joined is $\sim 1 / 100,000$. Whether it is just a coincidence that two fragments of 11q13 are joined together in one transgenome in our MA-T1a1 transfectants or whether myeov may potentiate $h s t$ transforming activity remains to be determined. Recent studies reported a role for myeov in gastric and colorectal cancer and may therefore support this assumption $(36,37)$.

Our data also show that the interpretation of DNA transfection results may not always be definitive, especially 
if the gene under scrutiny does not possess any transforming capability in NIH/3T3 cells. In these cases a thorough investigation, such as a sequence analysis, of all the human material present in a tertiary transfectant should be performed.

\section{Acknowledgements}

We thank Hiromi Sakamoto, Genetic Division, National Cancer Research Institute, Tokyo, Japan, for kindly providing us with the pORF1 plasmid containing an hst (fgf4) cDNA fragment.

\section{References}

1. Janssen JW, Vaandrager JW, Heuser T, et al: Concurrent activation of a novel putative transforming gene, myeov, and cyclin D1 in a subset of multiple myeloma cell lines with $\mathrm{t}(11 ; 14)(\mathrm{q} 13 ; \mathrm{q} 32)$. Blood 95: 2691-2698, 2000.

2. Brecht M, Steenvoorden AC, Collard JG, et al: Activation of gef-h1, a guanine nucleotide exchange factor for RhoA, by DNA transfection. Int J Cancer 113: 533-540, 2005.

3. Janssen JW, Steenvoorden AC, Schmidtberger M and Bartram CR: Activation of the mas oncogene during transfection of monoblastic cell line DNA. Leukemia 2: 318-320, 1988.

4. Janssen JW, Schulz AS, Steenvoorden AC, et al: A novel putative tyrosine kinase receptor with oncogenic potential. Oncogene 6: 2113-2120, 1991.

5. Janssen JW, Braunger J, Ballas K, et al: Spectrum of transforming sequences detected by tumorigenicity assay in a large series of human neoplasms. Int J Cancer 80: 857-862, 1999.

6. Janssen JW, Cuny M, Orsetti B, et al: MYEOV: a candidate gene for DNA amplification events occurring centromeric to CCND1 in breast cancer. Int J Cancer 102: 608-614, 2002.

7. Janssen JW, Imoto I, Inoue J, et al: MYEOV, a gene at 11q13, is coamplified with CCND1, but epigenetically inactivated in a subset of esophageal squamous cell carcinomas. J Hum Genet 47: 460-464, 2002.

8. Sakamoto H, Mori M, Taira M, et al: Transforming gene from human stomach cancers and a noncancerous portion of stomach mucosa. Proc Natl Acad Sci USA 83: 3997-4001, 1986.

9. Delli-Bovi $\mathrm{P}$ and Basilico $\mathrm{C}$ : Isolation of a rearranged human transforming gene following transfection of Kaposi sarcoma DNA. Proc Natl Acad Sci USA 84: 5660-5664, 1987.

10. Delli-Bovi P, Curatola AM, Kern FG, Greco A, Ittmann M and Basilico C: An oncogene isolated by transfection of Kaposi's sarcoma DNA encodes a growth factor that is a member of the FGF family. Cell 50: 729-737, 1987.

11. Taira M, Yoshida T, Miyagawa K, Sakamoto H, Terada M and Sugimura T: cDNA sequence of human transforming gene hst and identification of the coding sequence required for transforming activity. Proc Natl Acad Sci USA 84: 2980-2984, 1987.

12. Delli-Bovi P, Curatola AM, Newman KM, et al: Processing, secretion, and biological properties of a novel growth factor of the fibroblast growth factor family with oncogenic potential. Mol Cell Biol 8: 2933-2941, 1988.

13. Peters G, Brookes S, Smith R, Placzek M and Dickson C: The mouse homolog of the hst/k-FGF gene is adjacent to int-2 and is activated by proviral insertion in some virally induced mammary tumors. Proc Natl Acad Sci USA 86: 5678-5682, 1989.

14. Jainchill JL, Aaronson SA and Todaro GJ: Murine sarcoma and leukemia viruses: assay using clonal lines of contact-inhibited mouse cells. J Virol 4: 549-553, 1969.

15. Bubenik J, Baresova M, Viklicky V, Jakoubkova J, Sainerova H and Donner J: Established cell line of urinary bladder carcinoma (T24) containing tumour-specific antigen. Int J Cancer 11: 765-773, 1973.
16. Janssen JW, Lyons J, Steenvoorden AC, Seliger H and Bartram CR: Concurrent mutations in two different ras genes in acute myelocytic leukemias. Nucleic Acids Res 15: 5669-5680, 1987.

17. Frischauf AM, Lehrach H, Poustka A and Murray N: Lambda replacement vectors carrying polylinker sequences. J Mol Biol 170: $827-842,1983$

18. Grosveld FG, Lund T, Murray EJ, Mellor AL, Dahl HH and Flavell RA: The construction of cosmid libraries which can be used to transform eukaryotic cells. Nucleic Acids Res 10: 6715-6732, 1982.

19. Rubin CM, Houck CM, Deininger PL, Friedmann T and Schmid CW: Partial nucleotide sequence of the 300-nucleotide interspersed repeated human DNA sequences. Nature 284: 372-374, 1980.

20. Chomczynski P and Sacchi N: Single-step method of RNA isolation by acid guanidinium thiocyanate-phenol-chloroform extraction. Anal Biochem 162: 156-159, 1987.

21. Shackleford GM and Varmus HE: Expression of the protooncogene int-1 is restricted to postmeiotic male germ cells and the neural tube of mid-gestational embryos. Cell 50: 89-95, 1987.

22. Goryshin IY and Reznikoff WS: Tn5 in vitro transposition. J Biol Chem 273: 7367-7374, 1998.

23. Park M, Dean M, Cooper CS, et al: Mechanism of met oncogene activation. Cell 45: 895-904, 1986.

24. Shih C, Padhy LC, Murray M and Weinberg RA: Transforming genes of carcinomas and neuroblastomas introduced into mouse fibroblasts. Nature 290: 261-264, 1981.

25. Martin-Zanca D, Hughes SH and Barbacid M: A human oncogene formed by the fusion of truncated tropomyosin and protein tyrosine kinase sequences. Nature 319: 743-748, 1986.

26. Noguchi T, Galland F, Batoz M, Mattei MG and Birnbaum D: Activation of a mcf.2 oncogene by deletion of amino-terminal coding sequences. Oncogene 3: 709-715, 1988.

27. Galland $F$, Stefanova $M$, Lafage $M$ and Birnbaum D: Localization of the $5^{\prime}$ end of the MCF2 oncogene to human chromosome 15q15----q23. Cytogenet Cell Genet 60: 114-116, 1992.

28. Janssen JW, Steenvoorden AC, Losekoot M and Bartram CR: Novel transforming sequences in human acute myelocytic leukemia cell lines. Oncogene 1: 175-179, 1987.

29. Sakamoto H, Yoshida T, Nakakuki M, et al: Cloned hst gene from normal human leukocyte DNA transforms NIH3T3 cells. Biochem Biophys Res Commun 151: 965-972, 1988.

30. Yoshida T, Sakamoto H, Miyagawa K, Little PF, Terada M and Sugimura T: Genomic clone of hst with transforming activity from a patient with acute leukemia. Biochem Biophys Res Commun 142: 1019-1024, 1987.

31. Schwab M: Amplification of oncogenes in human cancer cells. Bioessays 20: 473-479, 1998.

32. Hughes-Davies L, Huntsman D, Ruas M, et al: EMSY links the BRCA2 pathway to sporadic breast and ovarian cancer. Cell 115: 523-535, 2003.

33. Rodriguez C, Hughes-Davies L, Valles H, et al: Amplification of the BRCA2 pathway gene EMSY in sporadic breast cancer is related to negative outcome. Clin Cancer Res 10: 5785-5791, 2004.

34. Bekri S, Adelaide J, Merscher S, et al: Detailed map of a region commonly amplified at $11 \mathrm{q} 13-->\mathrm{q} 14$ in human breast carcinoma. Cytogenet Cell Genet 79: 125-131, 1997.

35. Ormandy CJ, Musgrove EA, Hui R, Daly RJ and Sutherland RL: Cyclin D1, EMS1 and 11q13 amplification in breast cancer. Breast Cancer Res Treat 78: 323-335, 2003.

36. Leyden J, Murray D, Moss A, et al: Net1 and Myeov: computationally identified mediators of gastric cancer. $\mathrm{Br} \mathrm{J}$ Cancer 94: 1204-1212, 2006

37. Moss AC, Lawlor G, Murray D, et al: ETV4 and Myeov knockdown impairs colon cancer cell line proliferation and invasion. Biochem Biophys Res Commun 345: 216-221, 2006. 